\title{
Sensory-Motor Activity as the Basis for Formation of the «Inner World» of A Person
}

\section{Vahram R Sargsyan*}

President of the International Academy of Neuroscience and Research associate L.A. Orbeli Institute of Physiology, Yerevan, Republic of Armenia

*Corresponding Author: Vahram R Sargsyan, President of the International Academy of Neuroscience and Research associate L.A. Orbeli Institute of Physiology, Yerevan, Republic of Armenia.

Received: September23, 2019; Published: October 15, 2019

DOI: $10.31080 /$ ASNE.2019.02.0118

\begin{abstract}
This scientific work proposes a new interdisciplinary approach in understanding the issue of the mechanisms of formation of small copies of society - the «inner world» of a person. The scientific basis for this was the new knowledge and achievements of modern neurogenetics and neurophysiology. The mechanisms of human sensory-motor activity for the formation of the human psyche and, as a consequence, the formation of society are presented. The value of research in its multidisciplinary approach to solving and correctly understanding the issue. Knowledge of the mechanisms of formation of society is very necessary and useful in a targeted and controlled formation of human society.
\end{abstract}

Keywords: Society; Sensory-Motor Activity; Man; Nano-Model Genetic Theory; Human Biocommunication System

\section{Introduction}

Society is a human community, the specificity of which is represented by the relations of people among themselves, their forms of interaction and association [3]. Here, interactions between people are ensured thanks to the sensorimotor activity of man. Unification is ensured due to the similar structure and functional activity of the biocommunication system [15] of society members and bio communicators $[12,18]$ that every healthy member of society inherited during ontogenies during the formation of their acquired genome. If there are certain defects in the formation of the human biocommunication system, such people cannot fully integrate into society. The presence of "incorrect" bio communicators in humans during the formation of the acquired genome subsequently leads to the appearance of autistic children [13], who are also not adapted and poorly integrated members of society. Children isolated from society from the moment they are born will also have great difficulty integrating into human society, or they will not be able to become part of society at all. Here everything depends on the duration and degree of isolation of the child. This is due to the incorrect and defective formation of the acquired genome of this child.

For a complete understanding of the formation of society it is very necessary to know about such concepts as the sensory system of a person, human motility and speech. The following is a generally accepted knowledge of these terms.

Classical science notes that the sensory system is a combination of peripheral and central structures of the nervous system responsible for the perception of signals from the surrounding or internal environment. The sensory system consists of receptors, neural pathways and brain regions responsible for processing the received signals. The most famous sensory systems are sight, hearing, touch, taste and smell. Using the sensor system, you can feel physical properties such as temperature, sound, taste, or pressure. Also called sensory systems are analyzers. Analyzers are a set of formations that perceive, transmit and analyze information from the surrounding/internal environment of the body. 
Motility - motor activity of an organism or individual organs. Motility is understood as a sequence of movements that, in their totality, are needed to perform a specific task. Distinguish between large and small motility, as well as the motility of certain organs.

Speech is a historically established form of communication between people through language constructs created on the basis of certain rules. The process of speech involves, on the one hand, the formation and formulation of thoughts using linguistic (speech) means, and on the other hand, the perception of language constructions and their understanding. Speech is considered verbal communication. Thus, speech is a psycholinguistic process and an oral form of the existence of human languages.

The society in the phenomenological understanding is the set of social worlds of our mentalities, worlds embodied in our consciousness. Here the most interesting part already begins - the functional activity of bio communicators according to the principle of a nano-model theory of genome functioning. This is how the "inner world" of a person is formed, which is a small copy of the "common world", and of which society is a part.

So, we will present specific mechanisms of human sensorymotor activity activity for the formation of society. The value of research in its multidisciplinary approach in solving and correctly understanding the issue. Knowledge of the mechanisms of formation of society is very necessary and useful in a targeted and controlled formation of human society. This is precisely the practical significance of this scientific work.

To fully understand the scientific meaning of this scientific article, it is necessary to have certain knowledge about new viral theories, genome classification, nano- model theory of genome functioning, brain plasticity and genome plasticity, and the formation of higher human nervous activity. Therefore, we are forced, referring to our previous work, to briefly repeat the statements of the above. In addition, we recommend that readers follow the links in the list of references to our previous scientific works and familiarize themselves with them in more detail.

\section{New biological theories}

As a result of scientific meta-analysis, the viral theories presented in table 1 were created $[12,18]$.

According to generally accepted notions, a virus is a non-cellular infectious agent that can be reproduced only inside living cells (prokaryotic or eukaryotic). For more than 100 years, viruses have been considered one of the greatest mysteries of biology. Viruses infect all types of organisms, from bacteria, plants to animals and humans $[1,7,10]$. They are found in almost every ecosystem on Earth and are the most numerous biological form. Many researchers believe that viruses are obligate parasites, because they are not able to multiply outside a living cell [21].

\begin{tabular}{|l|l|}
\hline 1. & The Viral Theory of The Electromagnetic Reception \\
\hline 2. & The Viral Theory of Biocommunication \\
\hline 3. & The Viral Theory of Signal Transduction \\
\hline 4. & $\begin{array}{l}\text { The Viral Theory of Functioning of The Energy System of } \\
\text { Cell }\end{array}$ \\
\hline 5. & The Viral Theory of The Functioning of The Immune System \\
\hline 6. & The Viral Theory of Perception of Information \\
\hline 7. & The Viral Theory of Memory Formation \\
\hline 8. & $\begin{array}{l}\text { The Viral Theory of The Functioning of The Somatic Ner- } \\
\text { vous System }\end{array}$ \\
\hline 9. & $\begin{array}{l}\text { The Viral Theory of The Functioning of The Autonomic } \\
\text { Nervous System }\end{array}$ \\
\hline 10. & $\begin{array}{l}\text { The Viral Theory of The Functioning of The Endocrine Sys- } \\
\text { tem }\end{array}$ \\
\hline 11. & $\begin{array}{l}\text { The Viral Theory of The Functioning of The Cardiovascular } \\
\text { System }\end{array}$ \\
\hline 12. & $\begin{array}{l}\text { The Viral Theory of The Functioning of The Reproductive } \\
\text { System }\end{array}$ \\
\hline 13. & $\begin{array}{l}\text { The Viral Theory of Evolution of The Organic World and } \\
\text { Homo Sapiens }\end{array}$ \\
\hline
\end{tabular}

Table 1: Viral theories of VR Sargsyan.

But given the current level of knowledge in various fields of science, there is a need to revise some fundamental ideas about the true functions of viruses in Nature. Therefore, we performed a meta-analysis of various reliable scientific data, starting mainly from 1892 [18].

For the first time, the existence of the virus was proved at the end of the 19th century (1892) by the Russian scientist D Ivanovsky. After his research on tobacco plant diseases, D Ivanovsky concludes that tobacco mosaic disease is caused by "bacteria passing through the bacteriological filter, which, however, are not able to grow on artificial substrates." A few years later, when studying cattle diseases, a similar filtered microorganism was isolated. 
In 1898, when reproducing the experiments of Ivanovsky, the Dutch scientist M. Beyerink called such microorganisms "filtering viruses."

It is necessary to understand the true place and functions of viruses in nature, and by their example it will be possible to study the fundamental foundations of life.

Thus, we have formulated 13 viral theories, each of which reveals one of the functions of viruses in nature (Table-1). However, in this scientific article we will not dwell on their description in detail and for an introduction to viral theories, see our previous publications $[12,14,18]$. Particular attention should be paid to the viral theory of information perception, the viral theory of memory formation and the viral theory of the functioning of the somatic / autonomic nervous system.

In previous published works $[12,14]$ we concluded that viruses are migratory organelles of eukaryotic cells. They are part of us - cellular life forms and perform numerous functions. Viruses are not independent life forms and this is evidenced by the general biological cellular theory.

In 1898, the Dutch botanist M. Beyerink actually coined the term "virus", as he called such microorganisms "filtering viruses". After 120 years (in 2018) [18], based on the foregoing, we proposed replacing the term "virus" with the term biocommunicator, which certainly corresponds more to their functions in Nature.

Below, we present our classification of the genome into the basic and acquired and new genetic theory.

Main and acquired genome

The genome is hereditary material contained in the cell of the body. The genome contains biological information necessary for building and maintaining the body. Most genomes, including the human genome, are built from DNA and RNA. There is also another definition of the term "genome", in which the genome is understood as the totality of the genetic material of a haploid set of chromosomes of a given species [4,22].

The main genome is the totality of all genes received by the body from the egg and sperm as a result of fertilization and this is the vertical transfer of genes [17].
The acquired gene is the totality of all genes received by the body during the embryonic and postembryonic periods from migrating organelles of cells in the form of DNA and RNA molecules. It is important to note that the acquired gene can also be formed on the basis of existing bio communicator genes under the influence, for example, of electrical processes that occur in the nervous system of the body (see the viral theories of information perception, memory formation and functioning of the nervous system about this) [18], which take place as a result of the activity of the sensory systems of the body. The formation of the acquired genome is also influenced by electromagnetic radiation of natural and artificial origin. It turns out that all changes occurring in the external and internal environment of the body cause changes in the acquired genome; those that are significant are stored in the reserves of the long-term memory of the body. This is already horizontal gene transfer [18]. The acquired gene is individual for each somatic cell. If the process takes place in gametes, endovirus genes can form, which is already inherited.

The role of bio communicators in unicellular prokaryotic organisms, for example, plasmids play in bacteria. However, they are not able to perform all the functions inherent in bio communicators. Plasmids carry out active horizontal gene transfer in prokaryotes, viruses are analogues of plasmids for eukaryotes. Bacteriophages (bacterial viruses) are not bio communicators (migratory organelles) of bacterial cells and this is indicated by the fact that they forcibly introduce their genetic material into the bacterium. Therefore, we believe that bacteriophages are bio communicators of various eukaryotic cells, which provide for the regulation of various biochemical processes in bacterial cells, as well as control their numbers.

According to the information on the acquired and main genome, a new definition of the term "phenotype" can be given. The phenotype is a manifestation of the totality of genes obtained by vertical and horizontal channels of gene transfer and the result of their interaction. Therefore, the phenotype is the expression of the genotype. However, combinatorial and mutational variability contribute.

The body throughout life - from the moment of fertilization of the egg to death, has the ability to change (enrich or destroy) its genotype due to an increase/decrease in the proportion of 
the acquired genome. This is accomplished by horizontal gene transfer. Information obtained by the body's sensory systems about the external and internal environment actively influences the enrichment or depletion of the acquired genome. As a result, the phenotype of the body changes. However, these changes affect only the genes of certain cells of the body. For example, cells of the central nervous system of humans or animals, the immune system or cells of the stomach change. If the changes affect the germ cells, then new signs and properties will begin to be transmitted from generation to generation.

So, using the new classification of the genome into the main and acquired, it is possible to achieve a complete understanding of various biological processes that occur at the genetic, cellular and organismic levels of organization, under normal conditions and with various pathologies [17].

Genome plasticity as a basis for brain plasticity

According to the foregoing, the organism's genom is an actively and dynamically changing system throughout ontogenesis, but in order to make this thesis more convincing, we give an analogy with the plasticity of the human brain.

Neuroplasticity is a property of the human brain, which consists in the ability to change under the influence of experience, as well as restore lost connections after damage or as a response to external and internal influences. It was previously accepted that the structure of the brain remains unchanged after it is formed up to 5 - 6 years $[9,20]$.

The discovery of the fact that thoughts are capable, even in old age, of changing the structure and functions of the brain, is a major achievement in the field of neurobiology over the past 400 years. For example, Norman Dodge offers a revolutionary view of the human brain [8].

The brain consists of interconnected neurons and glia cells. The learning process can occur by changing the strength of the bonds between neurons, the emergence or destruction of bonds, and also due to the process of neurogenesis. Neuroplasticity refers to the processes of the emergence (and / or destruction) of bonds and neurogenesis $[9,20]$.

During the $20^{\text {th }}$ century, it was generally accepted that the structure of the brain stem and neocortex remains unchanged after completion of formation in childhood. This meant that the learning processes there can proceed only by changing the strength of the bonds, while the areas responsible for the memory processes, which retain the ability to neurogenesis throughout life, are highly plastic. This view is changing under the influence of new research, claiming that the brain retains its plasticity even in old age.

Neuroplasticity can manifest itself at different levels, from cellular changes in the brain, up to large-scale changes with reassignment of roles in the brain, as a response to damage to specific departments. The role of neuroplasticity is widely recognized by modern science and medicine, and also as a phenomenon used in the development of new methods of training and restoration of damaged brain.

The idea of "plasticity" of the brain was first proposed by William James in 1890. However, it was not given any importance over the next 50 years.

One of the fundamental principles of neuroplasticity is the phenomenon of synaptic pruning. The brain is constantly undergoing a process of destruction and creation of connections between neurons.

Thus, scientists are a little late, but still came to the conclusion that there is neuroplasticity. Why not accept the fact about the plasticity of the genome - the processes of genesis / destruction of genes in the process of ontogenesis of the body? With the help of such an understanding of the fundamental biological processes, one can understand and explain the numerous processes taking place in Nature.

\section{New genetic theory}

According to the nano-model theory of the functioning of the genome, a DNA molecule stores biological information not only in the form of a genetic code consisting of a sequence of nucleotides, but also in the form of a spatial-structural organization. This means that the information component lies not only in the primary structure of the organization of DNA molecules, but also in structures II and III. These are actually peculiar biological nanomodels [17]. However, RNA molecules and, to some extent, protein molecules $[12,14]$ can also perform a similar function in nature.

DNA contains information about the structure of various types of RNA and proteins [2], but this does not mean that the DNA molecule does not have the ability to independently carry out numerous biological functions that ensure the life of biological systems.

Citation: Vahram R Sargsyan. "Sensory-Motor Activity as the Basis for Formation of the «Inner World» of A Person". Acta Scientific Neurology 2.11 (2019): 13-21. 
Almost all genes of a living system function on the principle of nano-layouts. However, based on the fact that many genes of the main genome are localized in the cell nucleus and must function in the cytoplasm or outside the cell, therefore Nature has created transcription and translation processes known to modern science. The protein has a bulk structure due to its II, III, and sometimes IV structure. It is known, for example, that a protein-enzyme has an active center, functioning on the principle of "key to the lock." Depending on its form, it will have a certain functional activity. A DNA molecule also has a II, and III structure, that is, it is not just a linear molecule consisting of deoxyribo-nucleotides $[2,6]$.

The whole point of transcription and translation processes is to create a copy of the nano-layout in the form of ribosomal RNA, transport RNA, or messenger RNA. In the case of m-RNA, the biosynthesis of the polypeptide chain follows - translation on polyribosomes in the cytoplasm of the cell. In fact, ready-made copies of nano-DNA models capable of functioning outside the cell nucleus are provided. Protein biosynthesis is carried out as is known on the basis of m-RNA information, r-RNAs are part of ribosomes that are actively involved in protein biosynthesis, and t-RNAs are necessary for the delivery of amino acids to the site of protein synthesis. Many genes are composed of exons - coding regions and introns - non-coding regions. During transcription from the gene, RNA is read that carries both exons and introns. During splicing, introns are excised, and exons cross-link and form mature m-RNA; Further, the polypeptide chain of the protein will acquire a spatial-structural organization and become a functional protein in a functional sense.

Thus, the process of generating copies of nano-models based on biological information embedded in DNA is briefly presented here.

However, it is important to consider that some of the genes, mainly "junk genes" and some genes of the acquired genome, do not need such intermediary processes. Therefore, there is no need for transcription, translation processes, because these genes must function either in the place of their localization (regulatory genes, for example), or they have the ability to leave the cell nucleus and cells. This applies to bio communicators which for effective functioning and transportation are covered with a protein shell (capsid). Along with bio communicators, there are transposons. Transposons are parts of the DNA of organisms that are capable of movement and reproduction within the genome. Transposons are also known as "jumping genes" and are examples of mobile genetic elements.

According to our conclusions, it is for this reason that most of the human genome is non-coding protein. This is actually the socalled "gene language".

The molecular basis of epigenetics is quite complex, despite the fact that it does not affect the primary structure of DNA, but changes the activity of certain genes. This explains why in the differentiated cells of a multicellular organism only genes necessary for their specific activity are expressed. A feature of epigenetic changes is that they persist during cell division. Most epigenetic changes occur only within the life of one organism. At the same time, if a change in DNA occurred in the gametes (sperm or egg), then some epigenetic manifestations can be transmitted from generation to generation.

The nano-model theory of the functioning of the genome fully reflects the numerous processes taking place both at the cellular and organism levels. It is important to know that genes that function on the principle of nano-layouts are a kind of copy of the macrocosm. Depending on the degree of reflection of the macrocosm at the cellular level, one can judge the level of quality of perception of information from the biological system (for example, humans). The following expression is known: "The brain is in the World, and the World is in the Brain", which is already becoming understandable and explainable.

\section{The formation of higher nervous activity}

If we take into account the fact that the cell has a basic and acquired genome, then many unresolved scientific issues and, first of all, aspects of the genetic development of the biological system become clear. It becomes clear how and by what molecular mechanisms differentiation of cells in multicellular organisms is carried out in the process of individual development. It is scientifically substantiated that the appearance of highly specialized functions in neurons of the human brain and the manifestation at the organism level of various functions of higher nervous activity.

Therefore, geneticists studying the human genome are struggling to find those genetic features that led to an increase in the brain and, possibly, its more efficient work. Special hopes were placed on the comparison of the human genome with the genome of the monkey (chimpanzee). This allowed us to immediately exclude from consideration $98 \%$ of the genome, which are identical in these 
biological species. Somewhere there, in the remaining 2 percent, the secret of human uniqueness is encrypted and it remains to understand where exactly and how.

Immediately after reading the genome of chimpanzees and humans, genetics began to study the "secret" of human uniqueness. Publications devoted to the identification of the genetic characteristics of Homo sapiens are appearing more often, and it seems that a little more will be revealed to science. And today, the viral and genetic theories we have proposed are capable of explaining all this scientifically and logically.

Human behavior and mental abilities are on a qualitatively different level compared to chimpanzees. It is reasonable to assume that these differences are primarily of a genetic nature.

As a result of serious research, scientists proved that at the origin of man there was no universal and large-scale accumulation of amino acid changes in the genes involved in the work of nervous tissue $[5,19]$.

But Man is nevertheless smarter than chimpanzees and has a larger relative brain size. "Apparently, the development of our mental abilities is encoded by a very small number of genes, by changing their sequence or expression level. These changes do not affect the averaged characteristics for all genes of the nervous system" $[5,19]$. To such conclusions came these researchers.

According to our proposed genome classification (basic and acquired), viral theories and nano-model theory, all this can be explained logically and scientifically. The thing is that many geneticists study only the main genome of the body, that is, genes derived from parent germ cells. However, for the functioning of highly specialized cells (such as, for example, neurons) - those genes that were received from parents by vertical transmission (as a result of the formation of a zygote) will not be enough. According to viral theories, for the full perception of information, the formation of short-term and long-term memory and the functioning of the nervous system, the body in the process of ontogenesis must additionally receive a certain set of genes by horizontal gene transfer. This normally occurs in the perinatal and postnatal periods of ontogenesis of the body. In order for most of the highly specialized cells in the human body to begin to fully fulfill their functions, it is not enough just to "turn on" certain groups of genes and "turn off" other groups of genes from the main genome. If everything was so simple, then genetics would long ago have found many human genes that are inherent only to humans and distinguish us, for example, from other animal species. The fact that a person is quite superior to other biological species in terms of their level of development is beyond doubt. And these differences are due precisely to the receipt of additional genes already in the process of individual human development. The main human genome only creates the prerequisites and favorable conditions for the implementation of this process, and this requires only a small number of genes. According to modern genetic research, this is what distinguishes us, for example, from chimpanzees in the main genome.

\section{Bio-communication system}

The biocommunication system (BS) [15] is present in all multicellular organisms, but it is developed to varying degrees in various biological species. Here we present the structure and functions of the human BS. To date, of all the biological species known to science, the most complex BS has man. Therefore, we chose it as the object of study. The cause of many diseases is the destruction or dysfunction of the human biocommunication system $[13,16]$. Neurodegenerative and oncological diseases are a vivid example of the destruction of the human biocommunication system.

The structure of human biocommunication system

The main components of human BS are:

- Biocommunicators (DNA / RNA-containing). Biocommunicators are migratory organelles of human eukaryotic cells.

- Microbiome - a set of diverse microflora genes of various ecological groups. The microflora of man includes several thousand species of fungi, bacteria, archaea and viruses (biocommunicators). This is the so-called «bacterial» human organ.

- The ventricles of the brain are cavities in the brain filled with cerebrospinal fluid.

- Spinal canal - a longitudinal canal located in the gray matter of the spinal cord, at the top it connects to the cavity of the fourth ventricle of the brain; below forms a kind of expansion in the form of the final ventricle.

- The glyphatic system is a cerebrospinal fluid pathway for eliminating the products of vital activity of tissues of the central nervous system in mammals.

- Blood and lymph vessels, nerves - this is the transport infrastructure for biocommunicators. 
- $\quad$ Biocommunicator terminals or viral terminals - the boundary part of the central department of human BS, which provides a link between the visible body of the body and the environment.

- Free-living microorganisms (including biocommunicators) microflora isolated from the physical and visible human body. They constitute the aura of man and are the physical and invisible body of man. Man is actually an ecosystem inhabited not only by cells that arose during zygote division, but also by numerous representatives of microflora.

\section{Departments of human BS}

- Central BS - bio communicators and microflora within the physical and visible body of the body, the ventricles of the brain, spinal canal, glyphatic system, blood and lymph vessels, nerves and terminals of bio communicators.

- $\quad$ Peripheral BS - free-living microorganisms (including bio communicators) microflora isolated from the physical and visible human body. They are the physical and invisible body of a person, as they carry biological / genetic information about this biological system.

\section{Functions of human BS}

The human biocommunication system performs many vital functions and is closely related to the nervous, endocrine, immune and other human systems. Anatomically, BS can be located within all other human systems. In fact, wherever there is an active biocommunicator, a biocommunication system can function.

The main functions of human BS known to date are presented below.

\section{The function of electro-magnetoreception}

Thanks to this function, remote communication with the environment is ensured. The main natural sources of electromagnetic radiation in nature are the Sun, the Earth and other celestial bodies. The human BS provides the connection of the human body with the indicated celestial bodies, provides the integration of man with the environment.

\section{The function of biocommunication}

One of the most important BS features. Thanks to bio communicators, effective communication occurs between different biological species in nature. This is a biological communication channel when information from one individual to another is already transferred in the form of ready-made biological nano-models, with DNA, RNA and protein molecules. The biocommunication function has two varieties - biological attack and biological-humanitarian support.

\section{The function of signal transduction}

Bio communicators within the physical body of a person ensure the smooth operation of many trillions of human cells and other biological species. It is known that signal transduction is provided not only by physical and chemical interactions between cells of a multicellular biological system, but also biologically - using bio communicators. Violation of this function leads to the formation of oncological and neurodegenerative diseases in humans.

The function of controlling the energy system of cells

Bio communicators are responsible for many energy processes that occur in human cells. Violation of this function leads to dysfunctions of the mitochondria and oxidative stress. This is one of the reasons for the formation of many diseases.

\section{The function of controlling the immune system}

Bio communicators actively control cells of the human immune system and microflora cells. This ensures the control and safe life of a person, and protection from aggressors from the external / internal environment.

\section{The function of higher nervous activity}

A very important function and is based on the perception of information, the formation of memory and the functioning of the human nervous system. In other words, the sensorimotor activity of man and all of the above occurs with the active participation of bio communicators.

The function of monitoring the activity of various body systems

Bio communicators control the functioning of the nervous system, endocrine, cardiovascular, reproductive, digestive, respiratory, excretory and other human systems. This ensures the integration of all parts into a single whole.

The function of human development

Bio communicators are responsible for the processes of plasticity of the genome of an organism and neuroplasticity. This is the reason for development and this creates the basis for the process of evolution of man and the whole organic world.

\section{Human biocommunication system segments}

The biocommunication system consists of the following segments:

1. The segment of the present is the area of human consciousness where his real actions take place. They are based on information from numerous receptors on the human body. 
2. A segment of the past - is responsible for the long-term memory of a person. He characterizes the personality of a person of his «I».

3. The segment of the future is the place where a person builds his action plans for the future.

\section{Conclusion}

After we clarified the numerous functions of bio communicators in Nature, learned about the new classification of the genome and the nano-model genetic theory of the functioning of the genome, the mechanisms of the formation of the "inner world" or the human psyche (small copies of society) become clear. In fact, viruses (bio communicators) are migratory organelles of eukaryotic cells, and they perform many vital functions. Bio communicators are essentially the basis of life. Thanks to the correct understanding of the new classification of the genome and our genetic theory of the functioning of the genome, the mechanisms of the formation of higher nervous activity have become clear. Now it's clear how the «inner world» of a person is formed, and this is of strategic and very important importance for understanding the processes in the formation of society. Since it is also very important for the formation of interpersonal relationships in human society. However, one should not forget about the enormous influence of the external environment (for example, society) in the formation of the human psyche. This is how feedback is provided, and therefore the «inner world» of each person should normally be a small copy of the general «big world».

To understand the mechanisms of society formation, our viral theories of information perception, memory formation and functioning of the human nervous system are of decisive importance [12], as this determines the neurogenetic and neurophysiological aspects of human sensory-motor activity. As noted above, knowledge of the mechanisms of formation of society is very necessary and useful for the purposeful and controlled formation of human society. Here, in fact, there is actually a genetic modification of humans [11].

Thus, we can conclude that humanity has been engaged in the production of genetically modified people for many centuries, but is not even aware of it. A striking example is the world education system (schools, universities and other educational institutions). The activities of various security services in training the military and agents can be considered an example of obtaining a genetically modified person. The preparation and transformation of humans by terrorist organizations to create terrorists is also one example of human genetic modification. In this regard, various religious and social organizations, the media, medical institutions and, of course, science have a great impact on human genetic structures. We are sure that the goal of science is to help human society to finally correctly understand the processes occurring in society and nature in order to overcome its fear of the future. This will certainly lead to an increase in the quality and average life expectancy of a person and will lead to the creation of a more stable and beautiful World.

\section{Bibliography}

1. Alan J Cann. "Principles of molecular virology". 5th edition 320 (2011).

2. Alberts B., et al. "Molecular biology of the cell". 4th edition. New York: Garvard Science (2002).

3. Bhushan V and Sachdeva D. "Fundamentals of Sociology". Pearson Education India (2012): 308.

4. Inge-Vechtomov SG. "Genetics with the basics of selection". - St. Petersburg: Publisher NL (2010): 718.

5. Khaitovich P., et al. "Parallel Patterns of Evolution in the Genomes and Transcriptomes of Humans and Chimpanzees". Science 309 (2005): 1850-1854.

6. Klug W., et al. "Essentials of Genetics". Ninth edition (2017).

7. Nicholas H Acheson. "Fundamentals of molecular virology". 2nd edition (2011): 528.

8. Norman D. Brain Plasticity: Stunning facts about how thoughts are capable of changing the structure and function of our brain (2010): 544 .

9. Purves Dale., et al. "Neuroscience". 2nd edition (2001).

10. Sally Roberts. "DNA tumour viruses: virology". Pathogenesis and Vaccines (2018): 264.

11. Sargsyan VR. "New Biological Theories as a Basis for Safe Receiving Genetically Modified Person". ACTA Scientific Neurology 2.8 (2019).

12. Sargsyan VR. "New Scientific Theories - The Base for Creating Perspective Methods of Treating Different Diseases". Journal of Neuroscience 3 (2019): 008. 
13. Sargsyan VR "Formation of Higher Nervous Activity in Human and Autism Spectrum Disorder". Acta Scientific Women's Health 1.1 (2019): 22-24.

14. Sargsyan VR. "Formation of Human Nervous Activity and New Biological Theories". Journal of Neuroscience 2 (2018): 004.

15. Sargsyan VR. "Human Biocommunication System and New Health Care System". Alzheimer's Research and Therapy 2.1 (2019): 000105.

16. Sargsyan VR. "Mechanisms of formation of oncological and neurodegenerative diseases on the basis of viral theory of signal transduction. Med Crave". Advances in Obesity Weight Management and Control 9 (2019).

17. Sargsyan VR. "The main and acquired genome. Nano-models theory of the functioning of the genome. "International Science Project”. Finland. 1 part №17 (2018): 8-13.

18. Sargsyan VR. "The true place and role of viruses in nature. Viruses are migrating organelles of cells. "International Science Project”. Finland. 1 part №17 (2018): 4-8.

19. Shi P., et al. "Did brain-specific genes evolve faster in humans than in chimpanzees?" Trends in Genetics 22 (2006): 608-613.

20. Squire L., et al. "Fundamental Neuroscience". Third Edition USA (2008).

21. Taylor D., et al. "Biology". Moscow: MIR (2006).

22. Zhimulev I. "General and Molecular Genetics". - 1. - Novosibirsk: Novosibirsk University Press 459 (2002).

Volume 2 Issue 11 November 2019

(C) All rights are reserved by Vahram R Sargsyan. 